\title{
PROBLEMÁTICADE LOS CENTROS DE INTERMEDIACIÓN DE TIERRAS AGRARIAS EN ESPACIOS PERIURBANOS. DISCUSIÓN A PARTIR DEL EJEMPLO VASCO
}

\author{
Juan Cruz Alberdi Collantes \\ Departamento de Geografía. Universidad del País Vasco \\ juancruz.alberdi@ehu.es
}

\section{RESUMEN}

Los Centros de Intermediación de Suelo Rural, también denominados Bancos de Suelo, se crean en España a lo largo de la década de los 80 pero son pocos los que fructifican, siendo el modelo asturiano el referente casi único durante más de dos décadas. Los últimos años están surgiendo nuevas iniciativas que, tomando como referencia la estructura legal y administrativa de Asturias, comienzan su andadura. Responden, sin embargo, a posibilidades y realidades diferentes, especialmente a la hora de acceder al recurso tierra, y, como consecuencia, los resultados también difieren. En esta investigación analizamos los Centros de Intermediación que se están creando en el País Vasco y cuyos primeros resultados apuntan la dificultad de movilizar suelo privado y la necesidad de dotarlos de tierras públicas para materializar proyectos de desarrollo agrario. Además, el ejemplo muestra el camino para desarrollar este tipo de iniciativas en áreas rurales próximas a la ciudad.

Palabras clave: desarrollo agrario, suelo rural, banco de suelo agrario, País Vasco, Caserío.

\section{ABSTRACT}

Land Management Centres, call Land Banking too, born in Spain during the eighties but two decades later the Asturias model is the unique which marks the way to reference for the other. Later there are growing new initiatives which are taking the law and administration

Fecha de recepción: enero 2014.

Fecha de aceptación: septiembre 2014. 
structure of Asturias. But their possibilities and reality are different, especially the access to the ground and, therefore, the results are different. In this paper we study the Land Banking Centres in Basque Country. The first results show the difficulty to move private land and, as consequence, the necessity to move public land to development farm projects. Besides, this example shows the way to development land banking models in rural areas near to the city.

Keywords: farm development, farm ground, land banking, Basque Country, Basque farm unit.

Esta investigación resume los resultados obtenidos en el largo proceso que, iniciado hace una década, ha finalizado con la creación de un centro de intermediación de suelo agrario para el País Vasco. Como consecuencia obtenemos un modelo de promoción agrario en espacios donde el suelo útil es reducido y sometido a una fuerte presión urbana.

Las propuestas de intervención en suelo agrario en esta Comunidad comienzan a tomar forma a partir de la aprobación del Plan de Incorporación de Jóvenes Agricultores del País Vasco, referente principal incluso una década después de las políticas de desarrollo del agro vasco. El documento tiene dos objetos prioritarios, garantizar la renovación en el seno familiar al frente de la explotación agraria, por un lado, e incorporar nuevo agricultores, por otro. Asegurar el acceso al disfrute del suelo agrario es prioritario y, por ello, con él se da inicio a una batería de medidas de arbitraje que confluirán una década después en el desarrollo de los centros de intermediación.

La peculiaridad del sistema territorial vasco, con un caserío próximo a un entorno urbano, condiciona que iniciativas de captación de suelo agrario desarrolladas en Comunidades y Estados próximos no funcionen y que el resultado sea un modelo de intervención diferenciado.

Este trabajo es la crónica una década de experiencias fallidas que han tenido como conclusión la propuesta de un patrón fundamentado en suelo público, un modelo parcial, de desarrollo limitado, útil sólo para el progreso de determinados subsectores pero el único viable en espacios periurbanos y un laboratorio para aquellas regiones que, queriendo promocionar el desarrollo de la actividad agraria, se topen con un medio con similares características.

\section{LA SITUACIÓN ESPERADA, LA DIFICULTAD DE OPERAR EN ESPACIOS PERIURBANOS}

En esta investigación partimos del supuesto de que las políticas de desarrollo agrario en espacios de fuerte presión urbana tienden a fracasar puesto que el precio de la tierra deja de depender de sus posibilidades agrarias para quedar definido por sus expectativas urbanísticas.

Del mismo modo, se tiene la convicción de que la única opción de progreso del agro se produce como consecuencia de una relajación de la especulación, bien por razones económicas, bien por intervención en materia de ordenación del suelo agrario, presentado incluso en estos casos unos resultados insuficientes. Los modelos que buscan promover el mercado del suelo agrario entre particulares o adquirir tierras para redistribuirlas o para crear nuevos proyectos de desarrollo agrario también tienen poca o nula incidencia en espacios próximos a la ciudad. 
El análisis de la bibliografía relacionada con los bancos de tierras no hace sino corroborar la dificultad que un centro de intermediación puede tener en espacios periurbanos. En Europa funcionan actualmente diferentes iniciativas de este tipo, desde la escala nacional a la local, fundamentadas principalmente en tierras de su propiedad (Van Dijk et Kopeva, 2006), originarias de nuevos suelos útiles, procedentes de sobrantes de concentración parcelaria o, en los países de la Europa del Este, por redistribución por parte del Estado de fincas a sus antiguos propietarios (Boliari, 2013).

Hay otras iniciativas dirigidas a adquirir fincas mediante su compra, algunas a escala nacional, como la SAFER (Société d'aménagement foncier et d'établissement rural) en Francia, o a escala regional, como en Baviera (Klare y Doll, 2000), apoyadas en importantes dotaciones presupuestarias.

Una tercera vía es la de aquellas iniciativas que se apoyan en cesiones de particulares que se acogen a programas de prejubilación, algunos apoyados en el desarrollo de las directivas Europeas desde principios de los noventa, como el ejemplo de Asturias (Maceda, 1996) y otros incluso anteriores, como en el caso Holandés (Van Den Brink, 1990).

En todos los casos, a pesar del origen diverso, las iniciativas tienen un denominador común, generar una bolsa de suelo agrario que será distribuida, normalmente en arrendamiento, entre los agricultores. Sin activos propios y de condiciones agrológicas adecuadas los bancos de suelo tienen un recorrido reducido, como en Andalucía (Pérez Serrano et al. 2010) o en Galicia (Bantegal, 2013) y dónde actúa de intermediario y garante entre particulares pero careciendo de un patrimonio a gestionar. En muchos de estos casos, simplemente, pasado un tiempo el programa deja de funcionar

Si a la dificultad de crear una crear una bolsa de suelo se le añade el problema de operar en espacios próximo a la ciudad y con escaso suelo útil el nacimiento de las iniciativas se complica. En referencia al caserío vasco, Ainz (2001) señala que «nuestra agricultura se encuentra inmersa en una zona industrial con gran apetencia de tierra para destinos no agrícolas». Las iniciativas que analizan la operatividad de los bancos de tierras en espacios periurbanos escasean. Algunas están dirigidas a preservar estos espacios de la especulación para ordenar un crecimiento urbano e industrial intenso pero no para promover desarrollos agrarios (Ding, 2007). Otras hacen referencia al escaso desarrollo que programas como el de jubilaciones anticipadas tienen en los municipios próximos a la ciudad (Maceda, 1996) o la imposibilidad de adquirir fincas en estos espacios a un precios razonable (Merlet y Levesque, 2007).

Contextualizando el ejemplo del País Vasco en un entorno más amplio, la posibilidad de contar con suelo público o con suelo privado gestionado directamente por la entidad de intervención es una condición insalvable a la hora de asegurar el éxito de este tipo de iniciativas. Focalizando en un entorno más cercano al área de estudio, los instrumentos de gestión que perviven y tienen cierto éxito son aquellos que cuentan con recursos económicos que les permiten acceder a la compra de suelo, además de contar con ventajas legales que les reconoce intervenir en procesos de adquisición de fincas, y estos ejemplos no funcionan en espacios periurbanos.

Temiendo, por tanto, un escenario muy desfavorable para el progreso de iniciativas de desarrollo agrario fundamentadas en la captación de tierra, ¿Qué modelo de intervención podemos plantear en el caso del País Vasco, teniendo en cuenta que estamos en una región 
de una clara impronta urbana? El objetivo de esta investigación es el de responder a esta pregunta, proponiendo unas líneas de actuación que operen de guía para modelos que se pretendan desarrollar en entornos próximos a la ciudad.

La metodología que, para responder a la cuestión planteada se va a desarrollar, se apoya en la consulta bibliográfica de fuentes publicadas, especialmente las iniciativas que en Europa y en España se han puesto en marcha, y en investigaciones previas y publicadas del autor del trabajo. La consulta de estudios no publicados, realizados habitualmente en el marco de un proyecto de carácter institucional y con información especialmente válida a la hora de entender el funcionamiento del mercado del suelo agrario en el País Vasco es otra de las vías trabajadas.

Realmente satisfactorios han sido los resultados obtenidos tras la realización de entrevistas cualificadas. La consulta a los agentes que han intervenido en iniciativas anteriores y a los que participan en los proyectos actuales es fundamental para valorar las acciones iniciadas y conocer las expectativas que tienen las figuras de intervención. A lo largo de la investigación iremos haciendo constantes referencias a las personas y a las instituciones a las que representan los entrevistados, que podemos distribuirlos en diferentes grupos. En primer lugar, aquellas personas que participan en programas próximos sobre las que se apoya el caso en estudio y que tienen una mayor trayectoria. Así, entrevistamos al Gerente del GAL de la comarca de la Sidra y al Director de la Escuela Agraria de Villaviciosa, en Asturias, a ruralistas del Departamento de Geografía de la Universidad de Santiago y al presidente de la Cámara Agraria de Hasparne y miembro del Consejo Agrícola del Departamento de Pirineos Atlánticos. En segundo lugar, a actores que han intervenido o participan actualmente en las distintas empresas de intervención y de desarrollo agrario que se han o se estén desarrollando en el País Vasco.

En la medida en que analizamos un proceso con diferentes iniciativas que se extienden durante una década distribuimos los resultados en dos apartados, el primero dedicado a describir los antecedentes, en los que se analizará qué ha funcionado y qué no en las iniciativas iniciadas en el País Vasco, y el segundo, recogiendo el modelo por el que la administración ha optado y analizando los resultados que está obteniendo. Cerramos la investigación con una reflexión y recomendación sobre el camino que ha de llevar la intermediación en el suelo agrario de este territorio, y por ende, en espacios sometidos a fuerte presión urbana

\section{EL ANTECEDENTE, EN EL PLAN DE JÓVENES AGRICULTORES DEL PAís VASCO}

El plan joven, aprobado el año 2004, se apoya en la necesidad de abordar con urgencia un proceso de relevo generacional que permita la supervivencia de la función. Las acciones que plantea van dirigidas a impulsar una política de instalación neoprofesional, potenciando la incorporación de nuevos y cualificados agricultores, en un intento de rejuvenecer la profesión y mejorar su eficiencia.

El Plan es consciente de que su éxito no dependerá exclusivamente de la cuantía de las ayudas concedidas sino también de su articulación coherente con otro tipo de actuaciones en el ámbito de la intervención en el mercado de tierras, del cese anticipado de la actividad, de la formación profesional, regímenes sucesorios, etc. Y las medidas que propone van encaminadas en esta dirección (Alberdi, 2005b). 
Se formulan distintas figuras que potencien el acceso al disfrute de la tierra por parte de los jóvenes, como la incorporación de medidas propias de la legislación francesa («apadrinazgo»), la promoción de fondos privados de pensiones o la utilización de pastos comunales y montes de utilidad pública para el joven. Pero si algo es resaltado es la necesidad de crear una entidad de gestión que regule y dirija actuaciones encaminadas a defender y procurar suelo rural.

Para los redactores del Plan Joven, a pesar de los obstáculos y dificultades a los que se enfrenta la puesta en marcha de un órgano de intervención de suelo rural en una región urbana, su función es fundamental para incorporar activos que necesitan una base territorial sobre la que desarrollar su actividad. Gestionando una bolsa de suelo disponible se abre la posibilidad de incorporar población de origen urbano y ejercer labores de captación de proyectos y personas en los cursos de formación.

\section{II.1. Las medidas propuestas fracasan en sus intentos}

Una década después de ser aprobado el Plan de Jóvenes agricultores la mayoría de las iniciativas dirigidas a disponer de suelo agrario no se han desarrollado a pesar de que desde las administraciones se han hecho esfuerzos para que se materializaran. Buena parte de las propuestas se apoyaban en el cese de la actividad de las personas próximas a la edad de jubilación, liberando suelo agrario que desde un fondo público pudiera ser destinado a otros agricultores, preferentemente jóvenes.

Apoyada en los programas de cese anticipado de la actividad, regulados por los diferentes reglamentos comunitarios desde el año 1992, Asturias comienza a captar suelo que distribuye entre los agricultores con el objeto de rejuvenecer y mejorar las explotaciones agrarias. Al limitar al cesante la posibilidad de retener tan sólo el 10\% de su heredad, el banco gestiona los sobrantes atendiendo a sus objetivos y de una manera transparente. El éxito de la iniciativa es innegable, convirtiéndose en su momento en un referente en el desarrollo agrario del Principado y del Estado ${ }^{1}$.

De la necesidad de hacer frente a la demanda de suelo, y del éxito de la iniciativa de Asturias, deriva el Decreto del Gobierno Vasco $n^{\circ} 166 / 2000$, tras la reforma de la llamada «Agenda 2000», que al regular las ayudas por cese anticipado, dice en su artículo 23-3: «Asimismo, las tierras que quedan libres podrán pasar a disposición de un organismo que se comprometa a transferirlas posteriormente a un cesionario que cumpla las condiciones para el cese anticipado». Esta iniciativa es apoyada explícitamente por el sindicato agrario ENHECOAG, que en su propuesta de promoción de un organismo público de intervención en el factor tierra para el sector agrario (2002) considera el ejemplo de Asturias y las prejubilaciones como el medio prioritario para iniciar las políticas de intervención en suelo agrario.

1 A lo largo de esta investigación analizamos los diferentes modelos que desde el año 1987 se han ido creando en España. Aunque el modelo de Aragón marca un hito (López Ramón, 1989) es la iniciativa de Asturias la única que realmente se desarrolla, apoyándose en las prejubilaciones (Maceda Rúbio, 1996) si bien es verdad que esta iniciativa se ha visto progresivamente mermada una vez que el Principado ha ido reduciendo el apoyo al programa de prejubilación (Machargo, 2004; Iglesias, 2010). En la actualidad los agentes agrarios del Principado que entrevistamos se refieren al estado de la iniciativa como «paralizada», achacando su fracaso a la falta de recursos e entendimiento político para acometer su activización. 
El organismo al que apunta el decreto del Gobierno Vasco no se crea hasta una década después. El programa de cese anticipado en el País Vasco pasa totalmente desapercibido. A pesar del interés de las instituciones, en el periodo 2000-2006, en Guipúzcoa sólo 17 explotaciones se acogen a este programa, y en Vizcaya $20^{2}$, generalmente cesantes que dejan en manos de sus hijos el usufructo del suelo o personas que más que agricultores que participan como titular o cotitular lo hacen como integrantes de cooperativas de horticultores y que más bien ejercen la función de asalariados (Murua et al., 2006). No hay interés por acogerse a programas de cese de actividad y ello teniendo en cuenta que no se les obliga a ceder la disponibilidad del suelo a una tercera entidad si no lo desean. En la programación del periodo 2006-2013 estas ayudas son ya retiradas.

Las claves del fracaso del programa de cese anticipado de la actividad y, por tanto, de la creación de programas de intermediación en el País Vasco, se recogen en una investigación que, con el objeto de dinamizar una oficina de suelo agrario, es dirigida desde las Asociaciones de Desarrollo Rural del Valle del Deba. Las Asociaciones entrevistan a un total de 656 caseríos, con el objeto de conocer cuales eran las necesidades de suelo agrario que a medio plazo se plantearían. Del estudio se obtenían varias conclusiones, entre otras, una descripción del funcionamiento del mercado de suelo agrario en el territorio (Debagoiena, 2007). Resumimos a continuación las líneas principales que, respecto a las necesidades de suelo agrícola se planteaban.

- Los responsables del desarrollo rural de estas comarcas resaltan el alto grado de envejecimiento de la mano de obra agraria y el abandono exponencial que en los próximos años se ha de materializar.

- El propietario no es partidario de ceder el uso del suelo aunque no quiere que éste pierda su uso agrario. Lo mantiene con una cabaña que va progresivamente reduciéndose y extensificándose. Tampoco es partidario de firmar ningún contrato en el que figure que otra persona utiliza su propiedad. Si no puede mantener el uso del suelo, cederá su disfrute pero asegurándose que éste esté en todo momento a su disposición. No quiere las garantías de una administración porque, entre otras razones, no se fía de ella.

- La investigación nos aproxima al modelo de arrendamiento que domina en estas comarcas. El estudio utiliza el término «garbitzealde» (a cambio de su limpieza) refiriéndose a aquellos terrenos cedidos, normalmente para prado o pasto, con la única condición de mantenerlos limpios y de cuya constancia no existen datos puesto que rara vez son declarados o recogidos en censos agrarios.

- Del mismo modo, entre los ganaderos el único suelo que es demandado es aquel que puede ser objeto de laboreo con maquinaria agrícola. Ningún grupo, ni si quiera aquellos que se dedican a actividades de pastoreo, demandan otro tipo de suelo. El suelo mecanizable es escaso y el propietario no necesita acudir a la intervención de terceros para su arrendamiento.

2 Por realizar un ejercicio comparativo, en Asturias, el años 2002, se acogieron a este programa un total de 3.631, la Comunidad Autónoma con mayor número de personas acogidas a esta iniciativa en España (COAG, Anuario Agrario 2003). 
- Por otro lado, los precios que la administración baraja para estos arrendamientos, que rondan entre los 100 y los $500 € \mathrm{Ha} /$ año y que se corresponden con las posibilidades agrarias del suelo y con los precios agrarios del mercado privado, no son atrayentes para los propietarios. Aquel propietario de suelo rural que ya no es ganadero responde con indiferencia e incluso con indignación ante una propuesta de este tipo.

Los Agentes de Desarrollo Rural observan que el único suelo que puede ser objeto de gestión por una figura de intervención va a ser aquel que ningún ganadero haya querido previamente, es decir, aquel que peores condiciones agrológicas y que menores posibilidades urbanísticas presenta. Las aportaciones de suelo privado, a priori, parecían insuficientes para que la Oficina de Intermediación se convirtiera en una herramienta de dinamización de suelo agrario.

La intención de intervenir en materia de suelo agrario en el País Vasco no es nueva. De hecho, el año 2005 la Diputación de Guipúzcoa anuncia la creación de una Oficina de Intermediación de Suelo denominada Etorlur. Sin un documento legal ni decreto que lo ampare esta oficina pone gran parte de sus esfuerzos en promover arrendamientos entre propietarios y agricultores. Contaba con diferentes dinamizadores que captaban suelo a arrendar y que ponían a disposición de los agricultores. Mediaron entre el año 2005 y el 2012 en cerca de un centenar de contratos y fracasaron por su escaso resultado. Además de ello, el tipo de suelo arrendado era, salvo excepciones, aquel que peores condiciones agrológicas presentaba y que, por tanto, carecía de interés para los agricultores de la zona (parcelas alejadas, de montaña, con fuerte pendiente... $)^{3}$. El propietario sólo recurría a Etorlur cuando no encontraba interesado en arrendar su suelo.

Las causas del fracaso de muchas de las iniciativas dirigidas a movilizar suelo son habituales en los entornos rurales. La desconfianza en la administración y el temor a la pérdida de la propiedad son habitualmente citadas en la bibliografía (Carbadillo, 2008; Corbelle y Crecente, 2008; Creed, 1999) y en las entrevistas que efectuamos a responsables que trabajan en estas áreas. Pero hay otras causas propias de espacios próximos a la ciudad. En estos entornos las prejubilaciones agrarias que suponen una cesión del disfrute del suelo a terceros fracasan, los entes de intervención en el precio del suelo agrario no actúan o figuras como el padrinazgo no funcionan en los cantones próximos a áreas periurbanas ${ }^{4}$.

3 En una de las entrevistas realizadas en esta investigación la persona encargada de dinamizar arrendamientos agrarios en el territorio de Guipúzcoa (Unzurrunzaga, 2012) señalaba que tan sólo se dirigen a ella aquellos propietarios que en sus alrededores no han encontrado persona alguna interesada en alquilar sus terrenos, generalmente suelos de vocación forestal o pastizal. Nada extraño, por otro lado, si tenemos en cuenta que las posibilidades agrológicas de las cerca de un millar de parcelas que Bantegal, ente gestor del suelo agrario en Galicia, ofrece, son más bien escasas. La gran mayoría son inferiores a una Ha. y con vocación de pastizal e incluso arbolado. Como consecuencia el número de arrendamientos ha sido reducido, no superior al 15\% del suelo ofertado (www.bantegal. es). Paul Carril (2013), profesor de la Universidad de Santiago confirma esta situación señalando que «las tierras ofertadas son a menudo más parcelas de monte que de pradera, poco interesantes para el ganadero local».

4 Germain Ezponda (2005), Presidente de la Cámara Agraria de Hasparne y miembro del Consejo Agrario del Departamento de los Pirineos Atlánticos nos señala en la entrevista que «La Safer no funciona en los cantones próximos a Bayona. Una vez que los precios de la tierra aumentan por la proximidad urbana la Safer no puede acceder a su compra. Su acción se suscribe a la zona interior del País Vasco y del Bearn». 
El ejemplo que analizamos es una muestra de un entorno agrario de espacios periurbanos, caracterizado por un precio del suelo muy alejado de sus posibilidades agrológicas, una tierra demandada por sus opciones urbanas y unas expectativas de revalorización del suelo que dificultan en extremo cualquier medida de intervención.

\section{II.2. Suelo público y orientado a la horticultura, el único que prospera}

La única línea del Plan Joven iniciada es aquella apoyada sobre suelos públicos, generalmente originarios de excavaciones y rellenos ${ }^{5}$. De hecho, la toma de conciencia de la pérdida de suelo productivo que la agricultura va a sufrir como consecuencia de la programación de una gran infraestructura como es el tren de alta velocidad, lleva a plantearse por parte de las instituciones la creación de una entidad de suelo rural que, aprovechando la envergadura de los movimientos de tierra que la obra a efectuar va a generar, adquiera suelo para su gestión (IKT, 2003).

El origen de la idea, al menos parcialmente, parte de las soluciones espaciales que desde las Asociaciones de desarrollo rural dan al problema de la falta de suelo hortícola. El concepto de agroaldea se aplica en el País Vasco a polígonos de parcelas acondicionados para el uso de actividades agrícolas, preferiblemente de titularidad pública y con una dotación urbanística común, cuya característica principal es su puesta a disposición de los agricultores demandantes bajo arrendamientos de cuantía normalmente simbólica ${ }^{6}$.

El arranque de estas agroaldeas viene provocado por la creciente demanda de suelo por parte de nuevos agricultores que deseen instalarse en el sector y agricultores profesionales que desean trasladar, reubicar o ampliar sus explotaciones. Las Agroaldeas en funcionamiento se corresponden con polígonos de parcelas agrícolas de cultivo intensivo profesional. Este modelo es el utilizado en los agroaldeas de San Marcos (Errenderia-Donostia), Asteasu, Arragua (Oiartzun), Astigarraga, Urnieta y Lezo. Consiste en polígonos de propiedad pública, divididos en parcelas de entre 4.000 y $10.000 \mathrm{~m}^{2}$, con infraestructuras generales comunes (luz, agua, accesos, cierres) y destinadas al cultivo de hortalizas, plantas o flor cortada, tanto en cultivo directo sobre el suelo, como en hidroponía o maceta y en invernaderos o al aire libre.

En relación al tipo de terreno que utilizan, estas agroaldeas se asientan bien sobre tierras cultivables de bajo valor agrológico (Asteasu, Astigarraga, Lezo) o bien sobre terrenos baldíos, como pueden ser rellenos de vaguadas (Oiartzun) e incluso áreas recuperadas de vertederos (San Marcos). A pesar de estos inconvenientes estas parcelas resultan de interés por su escaso valor para otras actividades productivas, por lo que la competencia de aprovechamiento es menor que en otros suelos.

5 Es difícil encontrar ejemplos en los que se usen los suelos originados como consecuencia de la realización de una infraestructura para completar la oferta de los centros de intermediación. Entre la bibliografía analizada tan sólo hemos encontrado referencias a esta fórmula en Baviera. El Gobierno Regional adquiere una amplia franja de suelo con la que compensa, en primer lugar, a los agricultores afectados, y destina el suelo restante a mejorar explotaciones agrarias (Klare y Doll, 2000)

6 A lo largo de esta investigación, desde el Plan Joven hasta la creación de los Centros de Intermediación, profundizamos en el estudios de los polígonos hortícolas, cuyos resultados se pueden consultar en Alberdi (2005a) y en toda una serie de artículos recogidos en la revista de horticultura de la Cornisa Cantábrica HAGINA, concretamente en los números $n^{\circ} 4,1995$, pp. 15-16; nº 12, 1997, pp. 36-41; nº 19, 1998, pp. 46-47; n² 20, 1999 , pp. 42-44. 


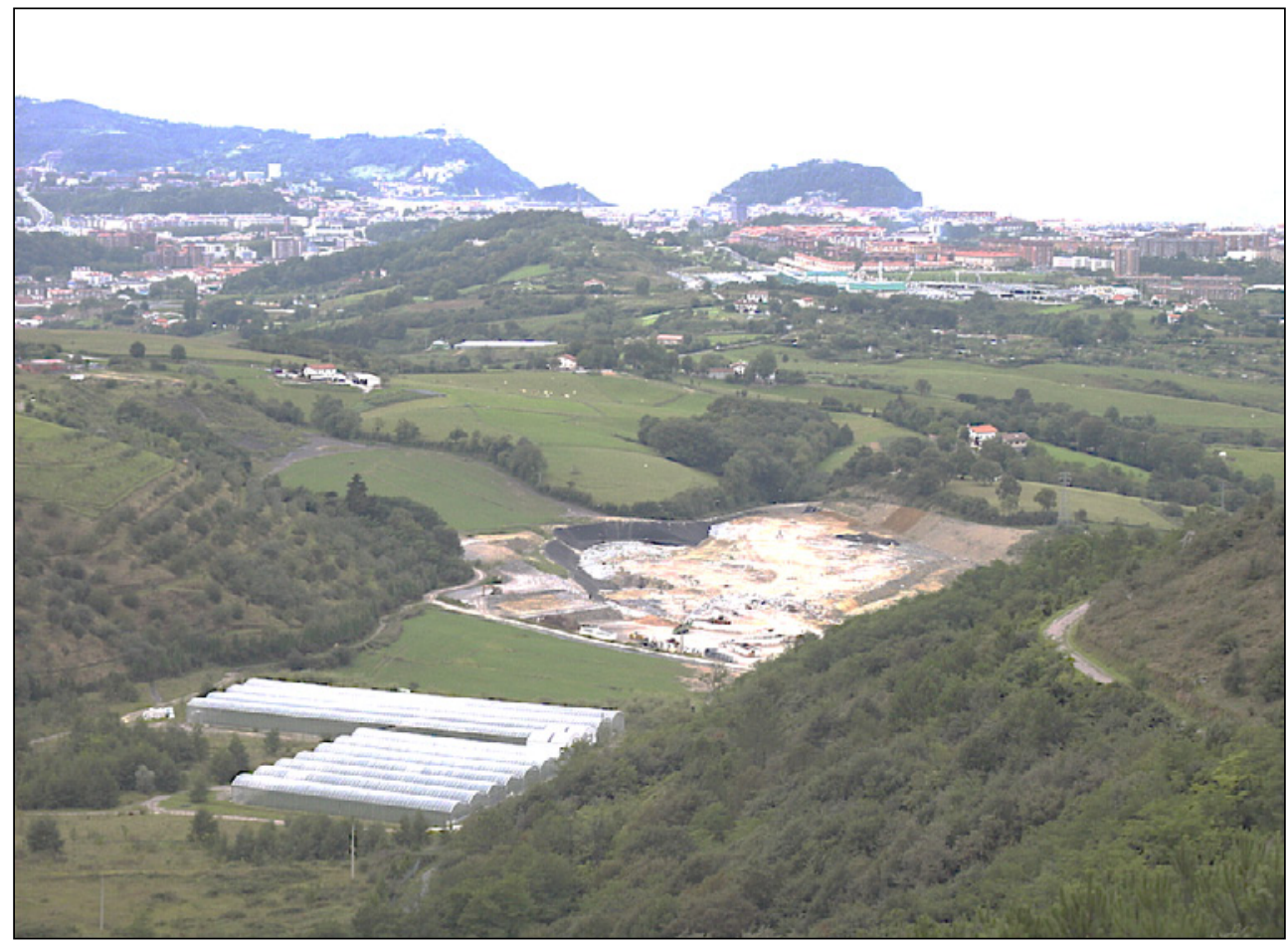

Desde el año 1996 en el que empiezan a desarrollarse los trabajos de impulso de la primera Agroaldea en Guipúzcoa se ha conseguido que sean ya siete los proyectos desarrollados, ampliación de 13 explotaciones preexistentes, creación de 7 nuevas explotaciones, implantación de $55.900 \mathrm{~m}^{2}$ de invernaderos, desarrollo de $15.500 \mathrm{~m}^{2}$ de explotación al aire libre y creación de 40 puestos de trabajo fijos y otros tantos temporales.

El modelo desarrollado, sin embargo, adolece de graves problemas originados por la propia debilidad de su constitución y que han quedado en evidencia con el paso de los años. El hecho de que la entidad que promueve la agroaldea no sea la propietaria del suelo y que éste continúe en manos municipales impide, ante conflictos por su utilización, que puedan reubicarse las explotaciones en terrenos adquiridos a partir de las plusvalías originadas en la venta del suelo que utiliza. En estos casos, con la agroaldea desaparecen las explotaciones instaladas en ella. Falta una entidad pública que gestione el suelo agrario y que aporte soluciones ante los problemas originados por conflictos de uso.

\section{UN CENTRO DE INTERMEDIACIÓN PARA ESPACIOS PERIURBANOS}

Tras los fracasos iniciales pero siendo conscientes de la necesidad de impulsar iniciativas que movilicen suelo agrario comienzan a crearse a partir del año 2012 los primeros centros 
de intermediación de suelo agrario en el País Vasco. La peculiaridad urbana del territorio, con las estructuras propias ya en funcionamiento, va a condicionar su evolución, incluso por derroteros que inicialmente no se preveían.

\section{III.1. El modelo elegido reproduce los centros de intermediación existentes}

En la actualidad en España hay dos iniciativas de gestión de suelo agrario promovidas por Comunidades Autónomas en funcionamiento, a las que se le une ahora el País Vasco y, previsiblemente, en breve tiempo Andalucia. Las primeras se crean a mediados de los 80 pero tan sólo Asturias desarrolla su proyecto las dos siguientes décadas (Maceda, 1996), apoyándose en los programas de cese anticipado de la actividad.

Los modelos de Banco de Tierras que se están creando en el País Vasco tienen su reflejo más inmediato en el Banco de Tierras que las Xunta de Galicia (Ley 7/2007, de 21 de mayo) pone en marcha en el año 2007 y coincide con el modelo organizativo que Asturias había diseñado.

Los medios utilizados para crear y hacer funcionar el banco de tierras en Galicia son tres: el Bantegal, las oficinas agrarias comarcales (OAC) y la Comisión de Precios (Carbadillo, 2008). En primer lugar, el Bantegal es la sociedad pública autonómica encargada de la gestión del Banco de Tierras. Por otro lado, se utiliza la estructura periférica ya existente de la Consejería del Medio Rural: las OAC, que ya actúan como enlace entre la Administración y el agricultor. A su vez, la Comisión de Precios surge ante la inexistencia de un mercado real de arrendamientos y ante la demanda de información sobre un precio objetivo de los alquileres.

El propietario o titular de una finca rústica o de una explotación agraria solicita la incorporación de ésta al Banco de Tierras como cesión temporal de usos. Así, queda en manos de la entidad realizar su explotación, así como el cultivo, mejora y otras actuaciones que considere necesarias. Una vez se incorpora una finca en el Banco de Tierras, éste tendrá la posibilidad de cederla en régimen de arrendamiento a terceras personas con fines agrarios por un período de cinco años o prórrogas sucesivas y períodos superiores, siempre con el consentimiento expreso del titular.

La aprobación por parte del Gobierno Vasco de la Ley 17/2008, de 23 de diciembre, de Política Agraria y Alimentaria, marca el primer paso en el proceso hacia la creación de entidades de gestión de tierra en el País Vasco. El desarrollo de la Ley, sin embargo, queda en manos de las Diputaciones Forales, aunque en este documento se definen las bases de su implementación así como el destino de los bienes y derechos que lo conforman, creándose para su gestión las denominadas oficinas de intermediación de suelo agrario.

A partir de la aprobación de la Ley, las diferentes Diputaciones ultiman sus propios decretos. Actualmente las tres diputaciones vascas han publicado su correspondiente decreto foral siguiendo los contenidos del documento legal del Gobierno Vasco ${ }^{7}$. Los decretos apenas difieren unos de otros y el esquema de funcionamiento que se plantea es similar al ejemplo de Galicia.

7 Decreto Foral de la Diputación Foral de Vizcaya 28/2010, de 9 de marzo, por el que se crea el Fondo de Suelo Agrario del Territorio Histórico de Vizcaya. Decreto Foral 10/2013, de 5 de marzo, por el que se regula el Fondo de Suelo Agrario del Territorio Histórico de Guipúzcoa. La Diputación Foral de Álava, por Acuerdo $\mathrm{n}^{\circ} 876$ del Consejo de Diputados de 9 de diciembre de 2008, procedió a la creación de una nueva sociedad pública, de capital cien por cien foral, con naturaleza jurídica de Sociedad Limitada y adscrita al departamento de Agricultura. 
La denominación que le aportan es la de «Fondo de Suelo Agrario» y su función principal es la de mediar con bienes y derechos de naturaleza rústica procedentes del sector público, de las fincas rústicas incorporadas por personas privadas y de aquellos procedentes de personas acogidas a ayudas de prejubilación cuyos suelos no tengan un destinatario predeterminado. El cometido queda en manos de la Oficina de Intermediación de Suelo Agrario, encargada de gestionar los bienes y derechos incorporados al Fondo.

Los objetivos también son análogos, entre los que destaca el asegurar la continuidad de las explotaciones agrarias, promover la incorporación de personas jóvenes, la permanencia y evolución en igualdad de condiciones de las mujeres del sector agrario, potenciar un dimensionamiento estructural de las explotaciones que coadyuve a su viabilidad económica y promover el desarrollo de una agricultura medioambiental sostenible, así como la transición agroecológica ${ }^{8}$.

Respecto a los aspectos propiamente agrarios se apuesta por una duración del arrendamiento que garantice la viabilidad de las inversiones que se han de realizar en la parcela. Éstas variarán según el uso que se le dé, más próximo a los 30 años en invernaderos y pabellones y más cercano a los 5 años en praderas y cultivos forrajeros. Los precios, fijados por una comisión interdepartamental, responden a las posibilidades agrarias del suelo y rondan entre los 100 y $500 € /$ año por Ha.

Las principales particularidades dentro de un esquema de funcionamiento similar se establecen en el territorio de Guipúzcoa. El reconocimiento explicito que se realiza de la presión de las infraestructuras urbanas sobre el medio rural condiciona parcialmente el decreto publicado a la hora de proceder a la adjudicación de las parcelas puesto que aquellas explotaciones profesionales sometidas a procesos de expropiación en las que su viabilidad quede condicionada tienen prioridad a la hora de acceder a éstas. Señala a su vez que, con carácter excepcional, y para proyectos de interés general, podrá realizarse la adjudicación directa de la cesión temporal de la finca a favor de Asociaciones de Desarrollo Rural (ADR) del Territorio Histórico de Guipúzcoa. Con ello se abre la posibilidad de desarrollar polígonos hortícolas en suelo público.

La gestión de los fondos de suelo rural en el País Vasco, por tanto, responde al esquema de funcionamiento habitual, desarrollado hace décadas en Asturias y actualizado en Galicia. Son sus peculiaridades administrativas, régimen foral, y la extensión del fenómeno urbano las que le aportan una impronta particular dentro de un escenario similar.

\section{III.2. Los primeros resultados: actuaciones públicas frente al desinterés del propietario}

Vizcaya es el primer territorio que pone en marcha una Oficina de Intermediación en el País Vasco, cuya gestión se apoya en la sociedad pública Basalan, creada el año $2010^{9}$. Con el objeto de informar al particular, realiza diferentes campañas dirigidas tanto a propieta-

8 Los objetivos apenas difieren de unos decretos a otros e incluso de unos estados a otros. Como señala Van Dijk (2003), el objetivo principal de los bancos de tierra desarrollados en Europa es la mejora de la explotación, preferenciando a aquellas que han sufrido algún daño (expropiación, concentración parcelaria,..) a explotaciones próximas, a jóvenes y a aquellas que poseen una formación adecuada.

9 Las políticas de intervención de suelo en la provincia de Alava se encontraban todavía en fase de proyecto a finales del año 2012. Aunque el marco legal y la sociedad de gestión pública están constituidas desde Abril de 1999, 
rios como a agricultores demandantes de suelo. Los resultados, sin embargo, no pueden ser más desalentadores. A pesar del interés mostrado y los esfuerzos realizados no se llega a materializar arrendamiento privado alguno. Los precios que se ofrecen no responden a las expectativas de los propietarios que prefieren optar por modelos de arrendamiento tradicional, es decir, contratos orales en precario generalmente sin contraprestación económica alguna ${ }^{10}$.

Tras varias campañas sin resultados se decide no promover más la vía del arrendamiento privado. Esta opción continúa habilitada pero no se realiza ningún esfuerzo adicional en la captación de tierras de origen privado. Tras esta decepción inicial desde las instituciones implicadas se es consciente de que la materialización del proyecto requiere de una intervención aún más directa, apoyada en suelos de titularidad pública.

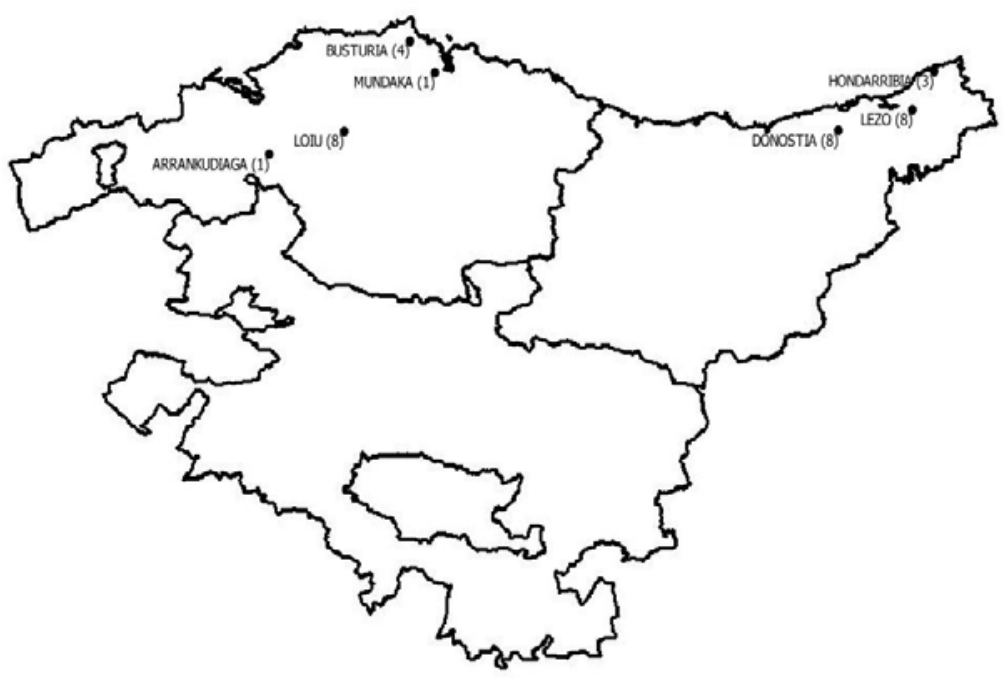

Fuente: Elaboración propia (datos en número de parcelas)

Así, la Diputación de Vizcaya, entre las primeras medidas adoptadas para activar el plan, adquiere una parcela de ochenta y cinco hectáreas aproximadamente en Busturia. La explotación, conocida como Zabalagoiko, cuenta con 33 Ha. de pradera, 20 de pastizal, 1,2 de huerta y $35 \mathrm{Ha}$. de monte. A su vez, las reparcela y realiza concesiones entre 5 y

en las entrevistas efectuadas a responsables de la Diputación Alavesa apuntan la intención de acometer este proyecto cara al próximo ejercicio si bien todavía a finales del 2013 no existía iniciativa alguna en marcha.

10 El desarrollo de este apartado coincide con el estudio de las primeras y únicas iniciativas que se están materializando. Ello nos lleva a que la entrevista a los responsables de las diferentes iniciativas sea la vía preferentemente utilizada para obtener información y valoraciones. En este caso se entrevista al responsable de Basalan, Enrique Aldekoa, al responsable de Gilur, Jacinto García, al reponsable de la ADR del área de Donostialdea y Bidasoa, Mikel Zendoia y a la responsable de la Agencia de Desarrollo Comarcal del Bidasoa, Aitziber Lacasta, además de a agricultores implicados en las iniciativas. 
30 años para desarrollar dos proyectos hortícolas, uno avícola y otro ganadero-forrajero. Los arrendatarios, todos menores de 45 años, desarrollarán su actividad a cambio de una renta establecida por la Comisión Técnica de Precios y Valoración, medida adoptada por la Diputación para evitar la especulación de precios, con rentas que van desde los 340 hasta los $600 €$ anuales.

La Diputación de Vizcaya, a mediados del año 2013 trabajaba en tres nuevos proyectos, todos ellos apoyados en suelo público. En dos casos, Mundaka y Arrankudiaga, arrendando parcelas municipales para uso ganadero. Y en un tercero, diseñando un polígono hortícola sobre suelos que el ayuntamiento de Loiu había adquirido para desarrollos residenciales y que, con el proyecto de ampliación del aeropuerto de Bilbao, habían quedado sin ejecutarse.

En el caso del territorio de Guipúzcoa ya se preveía que serían las intervenciones en suelos públicos las prioritarias tanto porque la disponibilidad de tierra es inmediata como porque dan la posibilidad de responder a una demanda cada más creciente de nuevos agricultores, de origen urbano y que carecen de suelo, y que quieren cultivar en ecológico. Así, la agroaldea de Urrullo, en el municipio de Lezo, es el primer proyecto de entidad que materializa el Centro de Intermediación y que no hace sino facilitar tierra a las entidades que ya anteriormente trabajaban con esta fórmula, en este caso, a la ADR de las Comarcas de Donostialdea y Bidasoa.

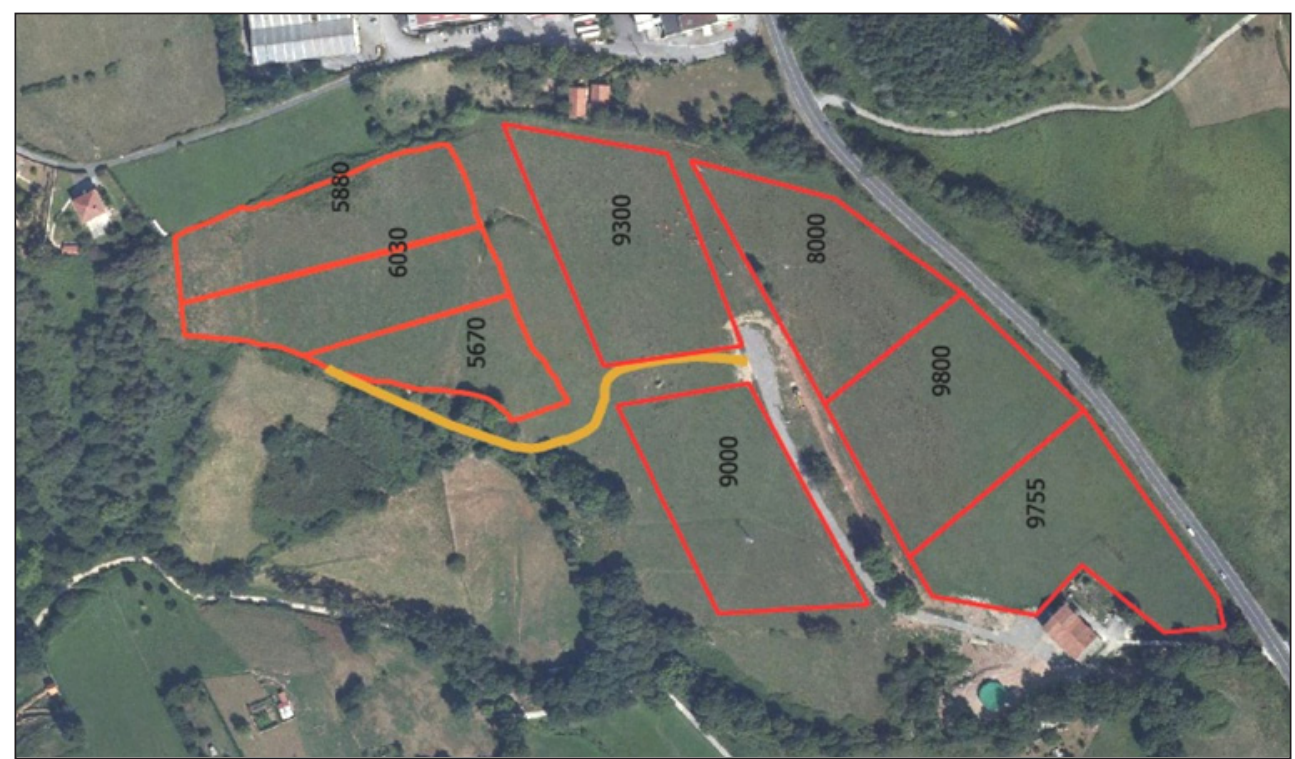

Fuente: ADR Behemendi. Elaboración propia (datos en $\mathrm{m}^{2}$ ).

En el diseño del proyecto confluyen dos elementos que lo hacen especial. En primer lugar, su localización estratégica en un área reservada al desarrollo del interland del puerto 
internacional de Pasajes, ahora descartada. En segundo lugar, su vocación ${ }^{11}$. La creciente demanda de producciones ecológicas y constante formación de grupos de consumo locales impulsa las producciones ecológicas.

Con las características descritas se instalan ocho explotaciones de cultivo ecológico de verduras y hortalizas en la parcela de Lezo, actividad compatible con el uso ganadero actual que todavía mantiene buena parte del área aprovechable. Cada parcela dispone de una superficie que oscila entre 0,6 y de 1 Ha., y combinan zonas de abono verde y barbecho. La superficie elegida es aquella considerada como necesaria para que un horticultor ejerza esta actividad a título principal.

Las mejoras realizadas, las necesarias para el progreso de la función hortícola digna, son las habituales en este tipo de intervenciones, es decir, contar con un vial de acceso a cada huerto ecológico, acometida de red de agua a cada parcela, construcción de almacén para aperos, verduras y hortalizas y preparación del terreno con volteado y abonado.

El propietario del terreno, la Diputación Foral de Guipúzcoa, cede la gestión del suelo a la Oficina de Intermediación, que a su vez realiza adjudicaciones por un periodo de 10 años, prorrogables anualmente, siempre y cuando ninguna de las partes implicadas lo denuncien. El funcionamiento y la gestión de las parcelas es supervisado por el Departamento de Desarrollo Rural de la Diputación Foral de Guipúzcoa y la Asociación de Desarrollo Rural, encargada de materializar y gestionar la agroaldea.

A la par que se ejecuta la agroaldea de Lezo se promueve el polígono hortícola de Botika. El anterior y el vigente Plan General de Ordenación Urbana de Hondarribia (Guipúzcoa), aprobado en Junio de 2012, prevé nuevos desarrollos residenciales en el barrio de Semisarga, en el área de Botika. Con este objetivo el Ayuntamiento de Hondarribia adquirió alrededor de $5 \mathrm{Ha}$. de caseríos de la zona. Estas proyecciones, sin embargo, han quedado paralizadas ante el descenso de la demanda y de los precios de la vivienda y que, como consecuencia, hacen inviables muchos de estos proyectos inmobiliarios.

Por otro lado, el desarrollo de la agricultura en el término municipal es una de las áreas que la ciudad ha incluido como prioritaria en el Plan de Desarrollo Local que, a instancia del Gobierno Vasco, ha desarrollado a lo largo del 2012. De hecho, la necesidad de materializar el proyecto surge de un curso de horticultura ecológica promovido por el ayuntamiento y apoyado por el INEM y en el que se observa que hay personas desempleadas interesadas en hacer de la horticultura su principal actividad. A raíz de la demanda el ayuntamiento decide dedicar los suelos de Botika a la ejecución de una agroaldea destinada a cultivo ecológico, cediendo las parcelas por un periodo de cinco años renovables ${ }^{12}$.

11 Según el informe de evaluación de los proyectos gestionados por la Oficina del Plan Del Joven Agricultor del País Vasco, Gaztenek, durante el periodo 2004-2012 en los territorios de Guipúzcoa y Vizcaya el 42\% presentaba una orientación hortícola, llegando esta orientación a ser próxima al 76\% en el caso de explotaciones de nueva creación. Su responsable en Guipúzcoa, Jesús Calvillo (2013), nos confirma que los últimos tres años en el País Vasco no se ha producido ninguna solicitud para producciones en invernadero de placa cuando años atrás había sido el modelo preferente para incorporarse al sector. Según él la crisis imposibilita realizar estas inversiones. Frente a ello, la crisis está potenciando producciones al aire libre y ecológicas, que no requieren apenas inversión y fácilmente asumibles por una persona parada que posee suelo. La horticultura, pero ahora en ecológico, sigue siendo la vía principal de incorporación al sector.

12 La organización del curso es realizada por la Agencia de Desarrollo del Bidasoa. Su responsable, Aitziber Lacasta (2013), nos comenta que «un total de 73 personas realizan la preinscripción para un curso al que solo podían acceder diez candidatos», una muestra del interés que, en un momento de crisis surge por esta nuevas activiades. 
La promoción de las últimas agroaldeas, junto a la demanda de suelo que en este momento se está produciendo, marca un cambio de tendencia respecto al tipo de productor que se había beneficiado de esta iniciativa. El modelo productivo fundamentado en el invernadero de placa, en el cultivo hidropónico y en la producción con label no está siendo atrayente, sea por falta de capital o por ideología, para el joven vasco.

Frente al horticultor profesional que ampliaba explotación o al joven que se iniciaba en un modelo productivista surge ahora un nuevo perfil de agricultor. De las diecisiete solicitudes presentadas sólo en cuatro casos lo hacen a modo individual, dominan los licenciados y la mayoría tiene una formación complementaria en horticultura. Los recursos económicos con los que cuentan son, sin embargo, muy reducidos. Muchos de ellos ya ejercía esta actividad anteriormente y algunos contaban con un canal de comercialización propio, como son los grupos de consumo ${ }^{13}$. Trabajaban suelos marginales, en condiciones difíciles, sin ningún tipo de cobertura, y ahora tienen la opción de hacerlo de una manera adecuada.

Figura 4

POLIGONO HORTÍCOLA DE BOTIKA. 2013

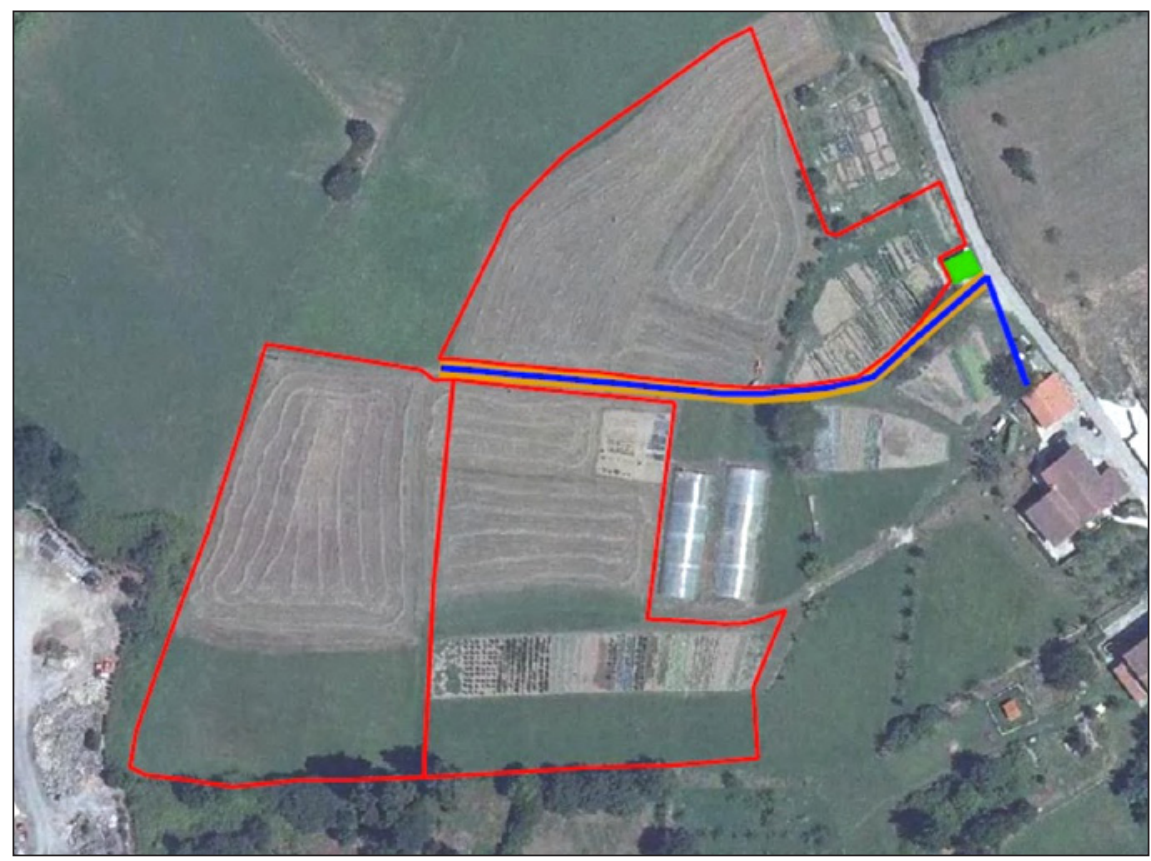

Fuente: ADR Behemendi. Elaboración propia.

13 En la entrevista realizada a Zendoia (2013), técnico de la ADR de Donostialdea, se nos describe el perfil del beneficiario de la agroaldea de Lezo y nos apunta que de los ocho adjudicatarios cuatro ya cuentan con un grupo de consumo. Es más, incluso dos de los aspirantes también están desarrollando esta vía de comercialización. Según Zendoia, en este momento falta producto ecológico local para abastecer la demanda de los grupos de consumo. El perfil coincide, al menos parcialmente, con el que en otras regiones se incorpora a la producción ecológica. Binimellis, Barceló y Canyelles (2004) analizan la tipología del agricultor ecológico de Mallorca, al que califican de joven, con estudios y con una visión de mercado diferente. 
El modelo descrito es el que se está desarrollando en otros ejemplos que comienzan ahora a proyectarse, en todos los casos sobre suelo público, destinado inicialmente a usos urbanos ahora desechados, y generalmente para cultivos ecológicos. Este es el caso de Antondegi, en San Sebastián, un área rural sobre la que se proyectó construir cuatro mil viviendas y para las que el Departamento de Vivienda del Gobierno Vasco compró un total de 28 Ha. hace ya 20 años. En este caso, la Oficina de Intermediación, el Gobierno Vasco y el Ayuntamiento ultiman un proyecto de desarrollo agrario que combina aprovechamientos ganaderos, hortícolas y frutícolas para el área.

\section{REFLEXIÓN: LA EVOLUCIÓN NECESARIA, DEL SUELO PÚBLICO A LA FISCALIZACIÓN DE LA TIERRA}

Las conclusiones obtenidas en esta investigación han de ser matizadas por el reducido tiempo que ha transcurrido desde la creación de los centros de intermediación pero a priori todo indica que el ejemplo iniciado en el País Vasco tiene unas posibilidades de progreso limitadas.

El análisis de modelos de gestión de suelo con mayor andadura permite contextualizar las opciones de éxito que puede tener la iniciativa que se está desarrollando en el País Vasco. Las posibilidades de progreso que pueda tener pasan por destinarle recursos económicos y, al menos hasta superar la actual coyuntura económica, este aspecto no se va a producir.

Otro aspecto prioritario es la actitud del propietario privado, que concentra la casi totalidad del suelo agrario, y que no acepta la intermediación pública salvo en aquellos suelos que peores condiciones agrológicas presentan y que no encuentran arrendadores en la proximidad.

El modelo desarrollado por los centros de intermediación agrarios en el País Vasco, al menos hasta el momento, se asienta sobre suelo público, en muchos casos destinado a usos urbanos y es de prever que agotará en un breve periodo de tiempo sus opciones de crecimiento. Al mismo tiempo, es un modelo de circunstancias, que responde a una situación de crisis, en la que suelos urbanizables aplazan su vocación y los agricultores son desempleados urbanos que han recurrido a la horticultura como medio de empleo. Una vez que las perspectivas mejoren la continuidad del modelo puede quedar en entredicho.

Los centros de intermediación del País Vasco, conocedores de sus limitaciones, tienen que profundizar en las peculiaridades de su entorno y buscar opciones de desarrollo que creen una iniciativa funcional y eficaz. Al mismo tiempo, han de desistir en ahondar en unas vías que en su territorio, al menos a medio plazo, no funcionarán. Entre éstas recogemos las siguientes:

- A diferencia de otras iniciativas no debe profundizar en intermediar entre particulares. Debe prepararse para afrontar esta situación pero sin realizar esfuerzos de dinamización. No se va a recurrir a su intermediación y si se hace va a ser a cuenta de suelo de escasas condiciones agrológicas, parcelas que no han encontrado agricultor interesado en la proximidad. Si se fundamenta sólo en este tipo de suelo, como lo han hecho otros, fracasará.

- El modelo que se asienta en el suelo cedido por prejubilación no funciona en entornos periurbanos. De hecho la administración vasca lo retiró ya en el anterior Plan de Desarrollo Rural y no lo recoge en los borradores que redacta en este momento sobre el PDR 2014-2020. 
- La opción más desarrollada por los bancos de suelo agrario de otras regiones europeas es la de generar suelo, para luego distribuir mediante arrendamientos a los particulares. Para ello se ha de contar con recursos propios (tierra) o adquirir suelo privado. Cada vez son más las fincas que se subastan y las entidades públicas bien pueden acceder a su compra. Incluso se pude estudiar la creación de un marco legislativo que garantice la presencia de sociedades de intermediación en las compraventas de suelo agrario (Merlet y Levesque, 2007). Pero los centros de intermediación vascos no tienen dotación presupuestaria, ni previsiblemente, la tendrán a medio plazo. ¿Cómo adquirir suelo sin presupuesto y más en un entorno en el que su precio real se aleja en exceso de sus posibilidades agrológicas y de las opciones de compra por parte de cualquier entidad?

Sin recursos propios ni posibilidades económicas es difícil plantearse el desarrollo de estas iniciativas, y menos en entornos donde el precio del suelo es tan elevado. Siendo conscientes de la dificultad llegamos a la conclusión que los esfuerzos en el País Vasco han de concentrarse en dos vías diferentes, aquellas que los recursos disponibles y la legislación le permiten.

La primera de las acciones es la de profundizar en la vía iniciada actualmente. Los entes forales tienen suelo en propiedad, al igual que ayuntamientos y el Gobierno Vasco. Es una bolsa reducida pero que ayuda a crear nuevos proyectos de desarrollo agrario en un territorio urbano. Ello genera expectativas en un área productiva deprimida. Provoca que la Oficina del Plan Joven Agricultor comience a movilizar proyectos de activos con problemas de suelo, que las ADR entablen relaciones con ayuntamientos, que estos entes mueven las redes de consumo locales... Atrae, a su vez, la atención de administraciones y departamentos que impulsan la iniciativa económica y que hasta ahora no habían mostrado interés por las posibilidades agrarias. De este modo se moviliza población activa, formación, recursos y ayudas económicas, además de suelo agrario.

El modelo impulsado, aunque no es exclusivamente hortícola, se centra en este subsector, el más demandado por el agricultor de origen urbano. Pero también es extensible a otro tipo de actividades que no necesitan mucho suelo, que promueven producciones de calidad y que tienen posibilidades de desarrollo, especialmente la avicultura con label y ecológica, y la fruticultura. Es un patrón que, dadas las circunstancias de crisis y de liberalización de suelo adquirido para otras actividades, por el momento se muestra suficiente para dar respuesta a la demanda de nuevos agricultores especializados en estas actividades. Sin embargo, se ha de tener presente que este camino es válido para impulsar una entidad de gestión de suelo en su inicio puesto que rápidamente agotará su recorrido sino viene apoyada de la movilización de suelo privado.

Junto a la dinamización de los activos propios, la otra vía a desarrollar es la movilización de suelo privado por infrautilización. Sorprende que en Vizcaya y Guipúzcoa no se haya intermediado en ningún arrendamiento privado. De hecho, el Gobierno Vasco, además de crear el Banco de Tierras, atiende al mantenimiento de la superficie agraria útil ${ }^{14}$. Con

14 El Gobierno Vasco publica el 8 de Octubre del 2012 el decreto Ley 193/2012 sobre promoción del uso y conservación del suelo agrario en el que regula el régimen sancionador por infrautilización del suelo agrario y en el que se habilita a las Diputaciones Forales a su desarrollo y aplicación. 
este objetivo, la ley establece mecanismos para la conservación y actuación sobre espacios infrautilizados, hasta el punto que prevé procedimientos sancionadores. La clave y el secreto del régimen sancionador de la ley es que las fincas que estén en el banco de tierras no sean multadas. Ello conlleva la cesión temporal de uso, por un plazo no inferior a diez años ni superior a treinta, a los fondos de suelo agrario de la parcela o parcelas implicadas.

Es evidente que si no se moviliza la propiedad privada los subsectores mayoritarios en este territorio, los ganaderos, no se van a beneficiar de las acciones de los centros de intermediación. Pero también es cierto que el propietario no acude a la mediación pública. El desarrollo del decreto de infrautilización y su materialización no va a traer más suelo a la entidad de gestión, no al menos de buenas condiciones agrológicas, pero va a movilizar los arrendamientos entre particulares. Mucho de la tierra mantenida en un régimen de subexplotación sería detectada (Corbelle y Crecente, 2008). El decreto del Gobierno Vasco le aporta hasta dos años para adecuar su explotación antes de proceder a su sanción. El propietario buscará ganaderos que quieran utilizar su heredad antes de verse sometido a un expediente sancionador y, aunque no acuda al centro de intermediación público, al menos movilizará su heredad.

El régimen sancionador de fincas mejorables no es una medida nueva en la legislación española. Andalucía ya la aplicó cuando aprobó su reforma agraria en 1984 y aunque tal reforma se considera un fracaso, al menos la legalidad de este tipo de actuaciones, tras resolver los recursos interpuestos, quedó solventada ${ }^{15}$. También Galicia y Asturias recogen la posibilidad de sancionar e incluso expropiar, pero en ningún caso se ha aplicado dado lo controvertido de las medidas. En el caso vasco, un incremento de las medidas impositivas a las fincas infrautilizadas y el inicio de un régimen sancionador a los casos más graves, movilizaría los acuerdos orales entre propietarios y ganaderos, beneficiando al propio banco de suelo y, sobre todo, al agro en general.

\section{BIBLIOGRAFÍA CITADA}

AINZ IBARRONDO, M.J. (2001): El caserío vasco en el País de las Industrias. Colección MAPA. Madrid.

ALBERDI, J.C. (2005a): Jóvenes agricultores: perspectivas, planes de dinamización y dificultades de instalación en el País Vasco, Papeles de Geografía. no 41-42, 5-28.

ALBERDI, J.C. (2005b): El polígono hortícola en el País Vasco como interpretación de las iniciativas territoriales industriales. Investigaciones Geográficas. nº 37, $27-74$.

BINIMELIS, J.; BARCELÓ, A. et CAYELLES, A. (2004): ¿Hacia una agricultura alternativa?. El desarrollo de la agricultura ecológica en Mallorca en el marco de la transición postproductivista. Geographicalia. n ${ }^{\circ}$ 42, 21-34.

15 La reforma agraria en Andalucía (1984) pretendía devolver la propiedad de la tierra a los obreros desposeídos (Gaviria, 1990) pero se topó con la oposición más frontal de los propietarios actuales del suelo. Ello le llevo a tener que sortear toda una serie de dificultades jurídicas consecuencia de los recursos interpuestos por particulares y grupos políticos que le retrasaron en su aplicación y le restaron eficiencia a pesar de que solventó legalmente la mayoría de los recursos que se le interpuso (Pérez Royo, 1992). El escaso suelo que enajena, generalmente fincas de concentración parcelaria, junto a aquel que era de su propiedad se subasta en diferentes lotes aunque éstos quedan generalmente vacantes (Pérez Serrano et al. 2010). El año 2010 el El Instituto Andaluz de Reforma Agraria (IARA), es disuelto. 
BOLIARI, N. (2013): Land Fragmentation in Bulgaria: Reconsidering Its Measurement and Extent. Review of Europan Studies. Vol. 5, n 1, 99-109.

CARBALLIDO PRESAS, X. (2008): La gestió dels espais agraris a Galícia: la Llei 7/2007 i el seu desenovolupament. La conservació de la superficie agrária útil i el banc de terres de Galícia. En La futura llei d'espais agraris a Catalunya. Jornades de reflexió, participació i debat. Anna Ribas Palom, Anna Roca Torrent, et al. (Ed.). 111-119.

DEBAGOIENA (2007): Herramienta de Gestión del Suelo Rural GILUR. Asociaciones de Desarrollo Rural del Valle del Deba. Etorlur. Documento Inédito.

COAG (2004): Estructuras agrarias y desarrollo rural. Cese anticipado de la actividad agraria. Anuario 2003. www.coag.org. Consulta 10-12-2013.

CORBELLE RICO, E. y CRECENTE MASEDA, R. (2008): El abandono de tierras: concepto teórico y consecuencias. Revista de Economía Gallega. n 2, 1-15.

CREED, G.W. (1999) The politics of agriculture: identity and socialist sentiment in Bulgaria. I Balcani-website.

DING, C. (2007): Policy and praxis of land acquisition in China. Land Use Policy, $\mathrm{n}^{\circ}$ 24, 1-13.

EHNE (2002): Organismo público de intervención en el factor tierra para el sector agrario. Euskal Herriko Nekazarien Elkartasuna. Lemoa (Vizcaya). www.coag.org. Consulta, 14-01-1013.

GAVIRIA, L. (1990): Reforma agraria y mercado de trabajo en Andalucia. Agricultura y sociedad. $\mathrm{n}^{\circ}$ 54, 267-293.

HAGINA (1993....): La revista Agrícola para la Cornisa Cantábrica. Oiartzun (Guipúzcoa).

HAZI (2012): Estudio del sector agrario en el Territorio histórico de Guipúzcoa. Diputación Foral de Guipúzcoa. Donostia. Inédito.

IGLESIAS. M.J. (2010) Medio Rural restringirá al máximo las prejubilaciones ganadera el próximo año. Publicación diaria digital. El diario de Asturias. Grupo Vocento. Gijón. Asturias. www.elcomercio.es. Consulta 18-9-2013.

IKT (2003): Oficina de Intermediación del Suelo Afectado por el trazado del la Y vasca. Gobierno Vasco. Inédito.

KLARE, K., DOLL, H. (2000): Private landwirtschaftliche Bodenfonds in Deutschland. Papers of the Institut für Betriebswirtschaft, Agrarstruktur und ländliche Räume der Bundesforschung sanstalt für Landwirtschaft (FAL).

MACEDA RUBIO, A. (1996): El banco de tierras de Asturias, con especial referencia a su gestión de los programas de cese anticipado de la actividad agraria. Ería. no 39-40, 115-142.

MARCHARGO, S. (2004): «PP denuncia la reducción de las prejubilaciones agrarias». Publicación diaria digital. El diario de Asturias. Grupo Vocento. Gijón. Asturias. www. elcomercio.es. Consulta 18-9-2013.

MERLET, M. y LEVESQUE, R. (2007): La SAFER, un mécanisme original de régulation des marchés fonciers par la organisations paysannes et L'Etat. AGTER. http://www. agter.asso.fr. (consulta 11-12-2013)

MURUA, J., EGUIA, B., MALAGÓN, E. y ALBIAC, J. (2006): El coste de la no agricultura en el País Vasco. Colección Lur. Gobierno Vasco. 284 p.

PÉREZ ROYO, J. (1992): Crónica Jurídica de la reforma agraria andaluza. Estudios Documento en PDF. www.navarra.es 
PEREZ SERRANO, J., VILLATORO, F., PEREZ SALINAS, M. y ROMÁN, A. (2010): La aportación de UGT Andalucía al medio rural andaluz. De la Reforma Agraria a el Desarrollo Rural (1980-2010). Fundación para el desarrollo de los pueblos de Andalucía. 254 p. PDF. Sevilla.

VAN DEN BRINK, A. (1990): Structuur in beweging: het landbouwstructuurbeleid in Nederland 1945-1985. Agricultural University Wageningen.

VAN DIJK, T. (2003): Scenarios of Central European land fragmentation. Land Use Policy, $\mathrm{n}^{\mathrm{o}} 20,149-158$.

VAN DIJK, T. y KOPEVA, D. (2006): Land banking and Central Europe: future relevance, current initiatives, Western European past experience. Land Use Policy, nº 26, 286-301. 